\title{
Dimensional analysis of partial discharge initiated by a metallic particle adhering to the spacer surface in a gas-insulated system
}

\author{
Firmansyah Nur Budiman*, Ali Muhammad Rushdi \\ Department of Electrical and Computer Engineering, King Abdulaziz University, PO Box 80204, Jeddah, Saudi Arabia
}

\author{
Article history:
}

Received: 29 September 2021 / Received in revised form: 7 December 2021 / Accepted: 13 December 2021

\begin{abstract}
Partial discharges (PDs) constitute important phenomena in a Gas-Insulated System (GIS) that warrant recognition (and, subsequently, mitigation) for being the obvious symptoms of system degradation. This paper proposes the dimensional analysis application based upon Buckingham $\pi$ theorem for characterizing PDs provoked by the presence of metallic particles adhering to the spacer surface in a GIS employing $\mathrm{SF}_{6}$ (Sulphur hexafluoride). The ultimate goal of the analysis is to formulate the relationships that express three PD indicator quantities, namely current, charge, and energy, in terms of six independent quantities that collectively influence these indicators. These six quantities (henceforth referred to as the influencing, determining or affecting variables) include the level of applied voltage, the $\mathrm{SF}_{6}$ pressure, the length and position of the particle on the spacer, the duration of voltage application, and the gap between electrodes. To compute the pertinent dimensionless products, we implemented three computational methods based on matrix operations. These three methods produced the precisely same dimensionless products, which were subsequently used for constructing the models depicting the relationships between each of the three PD dependent quantities and the common six determining variables. The models derived provide partial quantitative information and facilitate qualitative reasoning about the considered phenomenon.
\end{abstract}

Keywords: Partial discharge; GIS; dimensional analysis; dimensionless product; $\mathrm{SF}_{6}$

\section{Introduction}

Gas-Insulated Systems utilizing Sulphur hexafluoride $\left(\mathrm{SF}_{6}\right.$ GISs, for short) have been widely used for power substations worldwide, especially in densely-populated areas with very limited vacant spaces. The reason is that the dielectric strength of $\mathrm{SF}_{6}$ is much higher than that of air (typically three to four time), an advantage allowing a substantial compression of the substation size [1]. Moreover, the $\mathrm{SF}_{6}$ gas poses no dangerous detrimental threat to health since it enjoys the desirable feature of being both non-flammable and non-toxic. It also has good arcquenching properties and advanced heat transfer characteristics $[2,3]$.

However, any GIS cannot be optimally used to advantage with defects presence within it. One of the most notorious GIS defects is related to the presence of one or more metallic particles, which may be introduced into the GIS during manufacturing, installation, and/or operation. In certain conditions, such contaminants can adhere, stick, or attach to the insulation spac-

\footnotetext{
* Corresponding Author.

Email: fnbudiman@gmail.com

https://doi.org/10.21924/cst.6.2.2021.556
}

ers. If the electric field in the vicinity of the spacer is greatly enhanced, partial discharges (PDs) may increase, whose most inadvertent effect is the total breakdown of the insulators [4-6].

In some previous works [7, 8], Budiman et al. showed that PDs initiated by the presence of a contaminating particle in a GIS are heavily dependent on the particle length and its position on the spacer surface. Thus, the information regarding the PDs' characteristics has been utilized to estimate the particle length and its position. Budiman et al. also expressed concern in [9] that the presence of metallic particles inside a GIS can also cause the enhancement and the non-uniformity of the electric field around the GIS spacer.

The quantities related to (and acting as indicators for) the PD phenomenon in a GIS are obviously determined by a number of other factors. These affecting factors include the level of applied voltage, the $\mathrm{SF}_{6}$ pressure, the gap between the parallel-plate electrodes, and (possibly) several other factors. In the presence of a contaminating metallic particle, the length and the position of the particle are also among the affecting factors [8].

However, no aforementioned previous works have formulated quantitative relationships expressing the dependence of the 
PD quantities (indicators) on the affecting quantities. Most previous studies on PD analysis in GIS were mainly focused on how to recognize the PD pattern and to establish the PD model using the collected experimental data. This kind of research may utilize the conventional electrical signal [10], the VHF/UHF signal [11-16], and the accoustic signal [17] emitted by the PD. Other methods of measurement can also be utilized. However, AI-based pattern recognition techniques utilized in these studies were data-driven, in which they required sufficient data as the input, without which the recognition result was highly prone to errors.

Departing from the above mentioned gap, this study aimed to establish an appropriate mathematical formulation/model of the PD phenomenon in the GIS without a need for bulk experimental data. Instead of data-driven methods, we have to resort to an alternative adequate technique that might still provide the partial quantitative information and facilitate qualitative reasoning, without demanding so stringent theoretical requirements.

We asserted that dimensional analysis (DA) can serve for such a purpose, since all it needs is a proper identification of all the genuine determinants of the studied phenomenon together with the knowledge of the dimensions of the pertinent physical quantities with respect to an appropriate dimensional basis. Dimensional analysis is suitable for the exploration of phenomena that are not easily amenable to exact analysis. The examples of these phenomena include the estimation of the profit-loss sharing contracts [18], the modeling of aeolian sand bearing capacity [19], and the estimation of temperature at a point of an infrared dryer [20].

In power engineering-related studies, DA has not been extensively used. To the best of our knowledge, only few papers about DA in power systems can be found. The example of these few papers include [21, 22]. In [21], DA was applied for corona discharge analysis, but the insulating medium used was air and the electroce configuration was not specifically described. In turn, we have tried to give a detailed self-contained exposition of DA application to the considered phenomenon. We employed several matrix-based methods for DA implementation, and elaborated at its findings, relating them to the earlier experimental investigations.

The remainder of this paper is structured as follows. Section 2 describes the necessary materials and the methods used, emphasizing the basics of DA. Subsequently, Sections 3 presents a detailed exposition of DA application for our GIS problem. Our exposition covered three equivalent computational competitive (albeit equivalent) methods. Section 3 also provides a qualitative discussion and interpretation of the results obtained. Finally, Section 4 concludes the paper.

\section{Materials and Method}

\subsection{The Gas-Insulated System}

In this subsection, we would provide an ample description for the GIS system whose generated PDs are to be modeled herein, as discussed in [8]. This system is a laboratory-scale gas chamber whose schematic is shown in Figure 1. A spacer was located between the high voltage (HV) and the grounded electrodes, while a single small piece of metallic material (typically, referred to in the GIS literature as a particle) was glued on the spacer to replicate the condition in a real GIS, in which such a metallic particle is attached to the spacer. The level of the applied voltage was varied and various quantitative indicators of the generated PDs were measured at different $\mathrm{SF}_{6}$ pressure values by using a special PD measurement system, which is based on the IEC 60270 standard [23-26].

Note that the system shown in Figure 1 is a standard lab piece of equipment that mimics a real GIS. The use of this system was not meant as a restriction for the applicability of the developed PD model. Instead, we simply used the system to verify the parameters/quantities affecting the PD in a GIS. In other words, the developed model can be generalized to any real $\mathrm{SF}_{6}$ GIS.

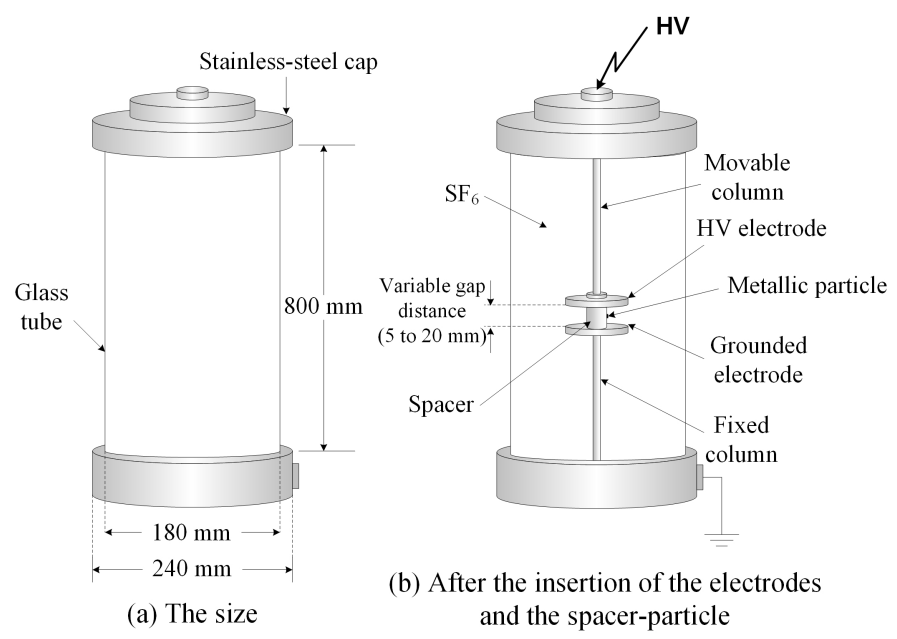

Fig. 1. The GIS chamber used for the experiment

In [8], Budiman et al. presented several PD distributions involving a number of PD quantities. Referring to these distributions, the PD quantities to be modeled in this study were selected to be the PD current $I_{P D}$, the PD charge $Q_{P D}$, and the PD energy $W_{P D}$. From the experiment conducted in [8], it was suggested that all these three quantities in the configuration used and under the prevailing circumstances were affected by the following six independent parameters/quantities: (i) applied voltage $V$, (ii) $\mathrm{SF}_{6}$ pressure $P$, (iii) particle length $L$, (iv) particle position relative to the grounded electrode $H_{p}$, (v) gap between electrodes/the height of the spacer $H_{s}$, and (vi) time/duration of voltage application $t$. Therefore, the mathematical relationship between each PD indicator quantity and these six parameters can be expressed as follows,

$$
\begin{aligned}
I_{P D} & =\Phi\left(V, P, L, H_{p}, H_{s}, t\right) \\
Q_{P D} & =\varphi\left(V, P, L, H_{p}, H_{s}, t\right) \\
W_{P D} & =\chi\left(V, P, L, H_{p}, H_{s}, t\right)
\end{aligned}
$$

Other important $\mathrm{SF}_{6}$ insulating parameters such as the dielectric constant (the relative permittivity), or the absolute permittivity itself were excluded in the current analysis since these two (proportional) quantities have been known to be dependent on the $\mathrm{SF}_{6}$ pressure value. There is a general trend that both of these quantities increase when the $\mathrm{SF}_{6}$ pressure gets higher $[27,28]$. Thus, the $\mathrm{SF}_{6}$ pressure level is deemed sufficient to represent each of them, i.e., to be used on behalf of any of them. This is a plausible assumption, indeed, that is much warranted in the present engineering approximation or our first cut at the 
problem, and might be subject to further scrutiny or verification at a later stage.

\subsection{Theory of Dimensional Analysis}

Dimensional analysis (DA) is an old, well-established yet powerful technique for qualitative reasoning and partial quantitative modeling. As summarized in [29, 30], DA enjoys several attractive features, including its capability to give a physical insight into the dependencies in physical systems, to simplify equations describing a system, and to unify seemingly disparate unrelated systems.

The fundamental paradigm of modern DA is established by the dimensional group's theorem, also known as Buckingham $\pi$ theorem, which states that an equation of $n$ physical variables can be reduced to another equation of $(n-r)$ new dimensionless variables, where $r$ is the rank of a certain matrix called as the dimensional matrix $[30,31]$. This matrix has some rows indexed by the physical dimensions used, columns indexed by the initial physical variables, and its typical element is the exponent to which the pertinent physical dimension (row index) is raised in a product expression of the pertinent variable (column index) [30]. The new variables or the dimensionless products constructed by DA are usually called as regimes [30].

If one has the following homogenous equation of $n$ physical variables,

$$
f\left(x_{1}, x_{2}, \ldots, x_{n}\right)=0
$$

then the reduced equation produced by DA is generally of the form

$$
F\left(\pi_{1}, \pi_{2}, \ldots, \pi_{n-r}\right)=0
$$

where the symbols $\pi_{i}$ denote the dimensional products or the new variables that replace the original variables $x_{i}$, and $F$ denotes an arbitrary function, whose determination lies outside the scope of DA. According to (5), any of these dimensionless products might be conveniently expressed as an arbitrary function of the others. If there is only one of these products $(n-r=1)$, then this single product is simply a constant, a situation in which DA is very effective, indeed. There are $(n-r)$ variables among the initial variables (called as regime variables), such that each of them appears in one regime only (with a unit exponent). If the dependence of a regime variable on other regime variables is ignored, then the value of its dimensionless product will be tentatively taken for a constant.

The initial task required to perform DA is to formulate the aforementioned dimensional matrix. The required procedure is presented as follows.

1. For all variables involved in modeling a certain system or characterizing a specific phenomenon, i.e., $x_{1}, x_{2}, \ldots, x_{n}$, their physical dimensions were formulated in terms of certain basic physical dimensions. For convenience, we used the MLT I dimensional basis utilizing the dimensions of mass $[M]$, length $[L]$, time $[T]$, and electric current $[I]$ as they are the four most prominent fundamental dimensions of the International System of Units (The SI System). Alternatively, we could have used the LTI $\phi$ basis, which replaces the dimension of mass by that of the electric potential or voltage $[\phi]$. Any of these two bases suffices to express all physical quantities incurred in the entire domain of electromagnetics (including kinetics, electricity, and magnetism as sub- domains). In more general problems, it might be convenient (albeit not strictly necessary) to supplement the dimensional basis by extra dimensions such as those of temperature, number of substances in moles, or illumination intensity (the remaining SI fundamental dimensions), as well as those of planar and solid angles (the auxiliary SI fundamental quantities).

2. Based upon Buckingham theorem [30], each dimensionless product constructed by multiplying each of the original variables (raised to a specific power) is of the form

$$
\pi=k x_{1}^{y_{1}} x_{2}^{y_{2}} \ldots x_{n}^{y_{n}}
$$

which can be converted into the following equation by taking the dimensions for all the quantities involved, namely

$$
1=[\pi]=\left[x_{1}\right]^{y_{1}}\left[x_{2}\right]^{y_{2}} \ldots\left[x_{n}\right]^{y_{n}}
$$

where $k$ is a dimensionless constant $([k]=1)$ and $y_{1}, y_{2}, \ldots, y_{n}$ are $n$ unknown (albeit related) exponents, which are yet to be partially determined. As seen shortly, some of these exponents are expressible in terms of the rest.

3. Another notation $p$ is defined, which represents the cardinality of the employed dimensional set, i.e., the number of basic physical quantities initially defined in the modeling. For the MLTI dimensional basis adopted herein, $p=4$.

4. The obtained dimensional equation in step 2 is converted into a set of $p$ equations in the following unknown exponents,

$$
\begin{gathered}
f_{1}\left(y_{1}, y_{2}, \ldots, y_{n}\right)=0 \\
f_{2}\left(y_{1}, y_{2}, \ldots, y_{n}\right)=0 \\
\vdots \\
f_{p}\left(y_{1}, y_{2}, \ldots, y_{n}\right)=0
\end{gathered}
$$

The set of equations in (8) is a purely homogenous linear set of equations in the unknown exponents, a fact that suggests its replacement by a single homogeneous matrix equation, involving a $p$-by- $n$ dimensional matrix $\Re(p<n)$ and a vector $\mathbf{Y}=\left[y_{1} y_{2} \ldots y_{n}\right]^{T}$ of unknown exponents, that is

$$
\mathfrak{R Y}=\mathbf{0}
$$

We temporarily assumed that the equations (8) are linearly independent; hence, the dimensional matrix $\mathfrak{R}$ is of full rank (has a rank $r=p<n$ ). However, if $r<p$, we replaced $p$ by $r$, and retained only $r$ linearly independent equations out of the set of equations (8). The literature abounds with techniques that assume that the rank $r$ is known from the outset (or assume it might be computed, say through computationally-intensive repeated determinant computation [32]). We pointed out that one of the computational methods suggested herein, namely the Gauss-Jordan method borrowed from [30], has the distinct advantage of not requiring a prior knowledge of $r$. This method proceeds towards its solution without making any distinctions between $p$ and $r$, and detects that $r$ is less than $p$ exactly according to the number of all-0 rows it generates. If no such rows are generated (mostly the case in small DA problems) then the assumption that $r$ and $p$ are the same is justified. Otherwise, $p$ is decremented by the number of all-0 rows generated, so as to represent $r$ correctly. As a bonus, the method of Gauss-Jordan 
elimination enjoys the additional advantage of deciding (with no extra computations) which $r$ out of the $p$ equations in (8) (i.e., which $r$ out of the $p$ rows of the dimensional matrix) to retain.

During this stage, both row interchanging and column interchanging may be recommended for shaping the first $p$ columns of the dimensional matrix to be as similar as possible to an identity matrix. The obtained matrix is to be taken herein as the designated dimensional matrix, which has a size of $p \times n$. Equation (9) is rewritten as (9a) to show the dimensional matrix structure, and to partition it into two matrices $\mathbf{A}$ and $\mathbf{B}$, the former of which is a square matrix.

$$
\left[\begin{array}{l|l}
\mathbf{A} & \mathbf{B}
\end{array}\right]\left[\begin{array}{c}
y_{1} \\
y_{2} \\
\vdots \\
y_{n}
\end{array}\right]=\left[\begin{array}{c}
0 \\
0 \\
\vdots \\
0
\end{array}\right]
$$

The size of $\mathbf{A}$ and $\mathbf{B}$ in (9a) are $p \times p$ and $p \times(n-p)$, respectively. Note that $(9 a)$ is written in the conventional form of writing a matrix equation. This form does not facilitate the reading of the constituent scalar equations, and might lead its reader to fallaciously assume that $p$ and $n$ are equal. Therefore, we will not abide by this conventional form when writing the detailed equations. Corresponding to the partitioning of the dimensional matrix, the set of unknown indices $\left\{y_{1}, y_{2}, \ldots, y_{n}\right\}$ in (9a) is partitioned into two disjoint sets [30]:

(a) A set of basis indices comprising the first $p$ indices, that is $\left\{y_{1}, y_{2}, \ldots, y_{p}\right\}$

(b) A set of regime indices comprising the remaining (n$p)$ indices, that is $\left\{y_{p+1}, y_{p+2}, \ldots, y_{n}\right\}$

The reasons of partitioning the dimensional matrix as shown in (9a), and the corresponding partitioning of the vector of indices will be clarified further as we proceed in this section. As previously mentioned, it is desirable to shape the submatrix $\mathbf{A}$ in (9a) to be as close as possible to an identity matrix. We noted that some authors have given higher priority to placing all variables required to act as the regimes in the submatrix $\mathbf{B}$ in (9a). These variables are also frequently referred to as output variables, since each of them appears only in a single dimensionless product. We will not insist on placing a variable destined to be a regime variable in the sub-matrix $\mathbf{B}$ in (9a). We will rely on a simple procedure (outlined in [30]) to restore any desired variable to a regime role.

Given a system with $n$ variables whose dimensional matrix is full rank, the number of possible sets of output variables is the number of selecting $(n-p)$ objects out of $n$ objects (with neither order nor repetition), which is $C(n, n-p)$ or $n$ choose $(n-p)$. This number equals the number of genuinely distinct ways of partitioning the dimensional matrix. If the dimensional matrix is not full rank, then this number becomes $C(n, n-r)$. Further elaboration about this point might be found in [30].

After the dimensional matrix was established, all dimensionless products $\pi_{i}$ were computed. Three methods were used in this paper for this purpose through the following steps.

\subsubsection{Method 1}

This method is based on the procedure outlined in [30]. The basic philosophy of this method is to integrate the determination of the rank $r$ of the dimensional matrix and the selection of $r$ linearly-independent equations with the solution process itself. To achieve this purpose, we applied the Gauss-Jordan elimination procedure, using row interchange when necessary to avoid pivoting on zero. In this procedure, the same elementary row operations were applied to the entire dimensional matrix [30, 33]. As a result, sub-matrix A was converted into a unit matrix, while sub-matrix $\mathbf{B}$ was converted into a sub-matrix $\mathbf{D}$, as shown in Figure 2.

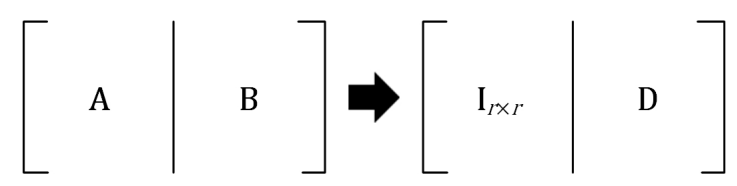

Fig. 2. Illustration of the dimensional matrix transformation via Gauss-Jordan elimination in which the same elementary row operations are applied to the entire matrix

The rank of the dimensional matrix $r$ is the size of the unit sub-matrix to which $\mathbf{A}$ is converted, while the size of $\mathbf{D}$ is $r(n-$ $r$ ). As stated earlier, $r \leq p$, so that either $r=p$ (for which case, it is acceptable to ignore the distinction between $r$ and $p$ ), or $r<p$ (for which case, $(p-r)$ all-0 rows emerge at the bottom of the processed matrix, and we ignored these rows by resetting $p$ to $r$ ). The values of the basic indexes $y_{1}, y_{2}, \ldots, y_{r}$ are then calculated in terms of the regime indexes as follows,

$$
\begin{gathered}
y_{1}=-d_{11} y_{r+1}-d_{12} y_{r+2}-\ldots-d_{1, n-r} y_{n} \\
y_{2}=-d_{21} y_{r+1}-d_{22} y_{r+2}-\ldots-d_{2, n-r} y_{n} \\
\vdots \\
y_{r}=-d_{r 1} y_{r+1}-d_{r 2} y_{r+2}-\ldots-d_{r, n-r} y_{n}
\end{gathered}
$$

where $d_{i j}$ are the elements of the matrix $\mathbf{D}$ and $i=1,2, r ; j=$ $1,2, n-r$.

The values of the basis indexes $y_{1}, y_{2}, \ldots, y_{r}$ from (10) were substituted into (6), while the remaining indexes (the regime indexes) $y_{r+1}, y_{r+2}, \ldots, y_{n}$ were left as they were. Afterwards, the quantities with the same exponent were grouped together as a single product until the following form was obtained,

$$
\pi=k \pi_{1}^{y_{r+1}} \pi_{2}^{y_{r+2}} \ldots \pi_{n-r}^{y_{n}}
$$

The dimensionless products $\pi_{i}(1 \leq i \leq n-r)$ were extracted from (11) by assigning orthonormal values to the elements of the set of regime indices $\left\{y_{r+1}, y_{r+2}, \ldots, y_{n}\right\}$, namely $\{1,0, \ldots, 0\},\{0,1, \ldots, 0\}, \ldots,\{0,0, \ldots, 1\}$. This meant that $(n-r)$ independent dimensionless products or regimes were obtained for this particular partitioning of the indices into basis and regime indices. Other independent set of dimensional products (with a specific required selection of regime variables) might also be obtained through an appropriate choice of $(n-r)$ independent points in the $(n-r)$-dimensional space of regime indices $y_{r+1}, y_{r+2}, \ldots, y_{n}$ [30]. Such points are deemed independent if none of them can be expressed as a weighted sum of the others. This means that we might deviate from the usual practice of selecting orthonormal points in the $(n-r)$-dimensional 
space of regime indices if we need a basis variable to act as a regime one. As seen shortly, each of the three output variables considered herein happened to appear in a single dimensionless product at no extra manipulation cost.

\subsubsection{Method 2}

This method, as explained in [29], is similar to method 1, but differs in the way the dimensional matrix is transformed as it uses a computationally less-efficient (albeit conceptually easier to grasp) procedure. We produced an $r$-by- $r$ unit matrix through left multiplication of the submatrix $\mathbf{A}$ by its inverse, that is

$$
\mathbf{I}_{r \times r}= \begin{cases}(\mathbf{A}[1: r, 1: r])^{-1} \mathbf{A}[1: r, 1: r] & r<p \\ \mathbf{A}^{-1} \mathbf{A} & r=p\end{cases}
$$

It is clear that the matrix $\mathbf{A}[1: r, 1: r]$ is non-singular as it is of full rank, and it might be identified as $\mathbf{A}$ when $r=p$. Similar to what was done in method 1 , operations implemented on submatrix $\mathbf{A}$ should also be done on sub-matrix $\mathbf{B}$, that is

$$
\mathbf{D}= \begin{cases}(\mathbf{A}[1: r, 1: r])^{-1} \mathbf{B}^{\prime} & r<p \\ \mathbf{A}^{-1} \mathbf{B} & r=p\end{cases}
$$

where

$$
\mathbf{B}^{\prime}=[\mathbf{A}[1: r, r+1: p] \mathbf{B}[1: r, 1: n-p]]
$$

Using the product of the two matrices $\mathbf{I}_{r \times r}$ and $\mathbf{D}$ transposed, together with a unit matrix $\mathbf{I}_{(n-r) \times(n-r)}$, we consructed a new matrix of the indexes used in the dimensionless products, as shown in Figure 3.

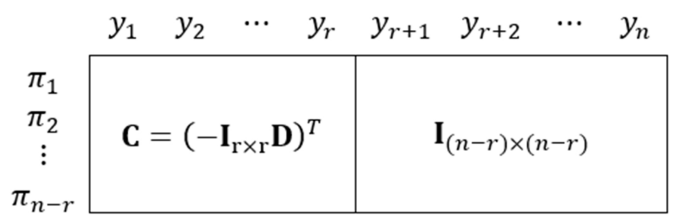

Fig. 3. Matrix constructed for $\pi_{i}$ calculation in the direct transformation method.

From the matrix in Figure 3, the dimensionless products $\pi_{i}$ were calculated as follows,

$$
\pi_{i}=x_{r+i} \prod_{j=1}^{r} x_{j}^{c_{i j}}
$$

for $i=1,2, n-r$, where $c_{i j}$ are the elements of submatrix $\mathbf{C}$.

\subsubsection{Method 3}

Method 3 is much similar to method 2, in which the target was still to create a matrix of the structure shown in Figure 3. The difference was that such a matrix was formed directly from submatrices $\mathbf{A}$ and $\mathbf{B}$, without undergoing the transformation illustrated in Figure 2.

As in method 2, prior knowledge of the rank of the submatrix $\mathbf{A}$ is required. Assuming $\mathbf{A}$ is non-singular and the rank of $\mathbf{A}$ is known,

$$
\mathbf{C}= \begin{cases}\left((\mathbf{A}[1: r, 1: r])^{-1}\left(-\mathbf{B}^{\prime}\right)\right)^{T} & r<p \\ \left(\mathbf{A}^{-1}(-\mathbf{B})\right)^{T} & r=p\end{cases}
$$

The dimensionless products $\pi_{i}$ were then calculated using (15).

\subsection{Summary of the Method}

The whole steps we used in establishing the PD model are summarized by the flowchart shown in Figure 4. Note that the workflow depicted in Figure 4 is for a single PD quantity. Therefore, it was repeated for all three PD quantities in (1)-(3).

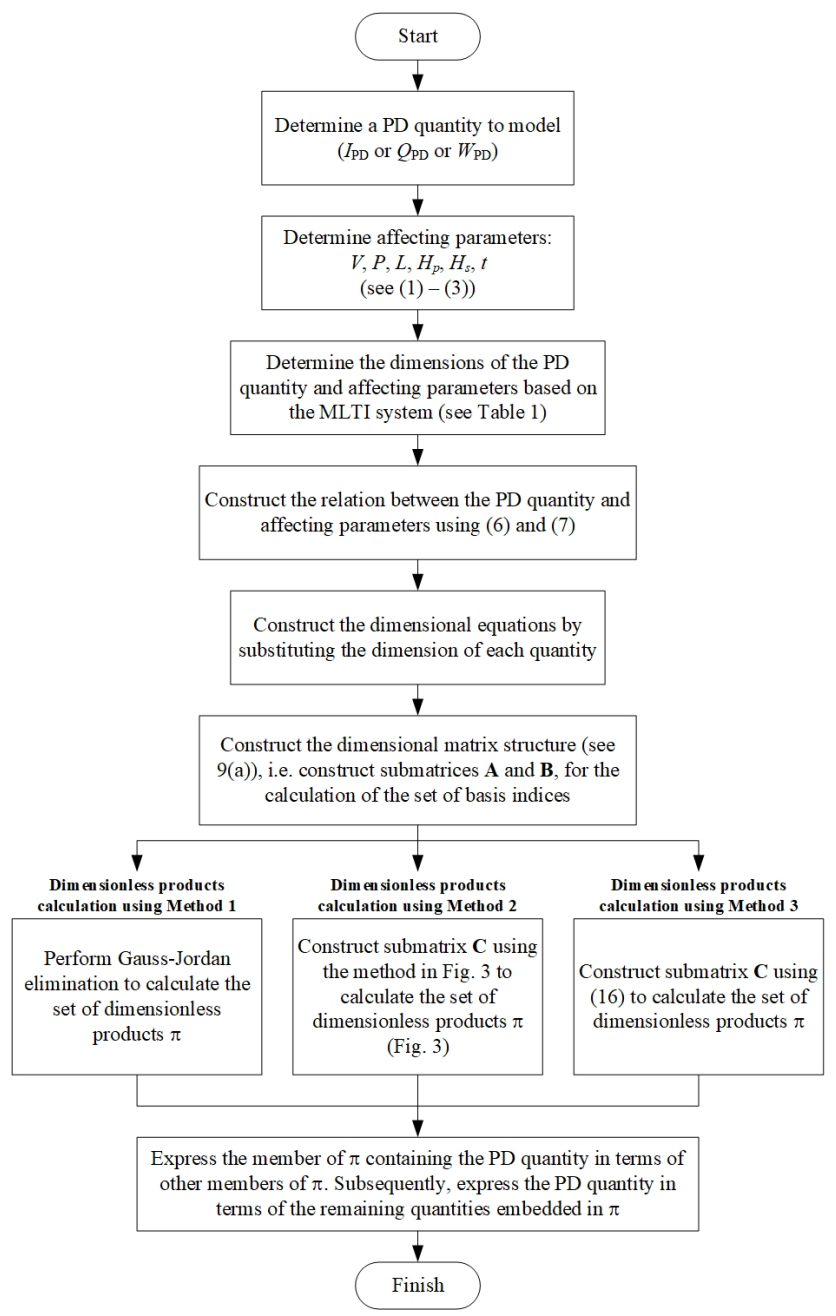

Fig. 4. Workflow that establishes the DA model of each PD quantity studied herein.

\section{Dimensional Analysis Application: Steps, Results, and Discussion}

In this section, we present the detailed operations to calculate the dimensionless products of the GIS variables to be interrelated by DA herein. These variables have been defined earlier in (1)-(3), and are listed again, along with their dimensions (within the MLTI dimensional basis), in Table 1.

Each of the first three indicator quantities in Table 1 is dependent on all the last six independent quantities and will be modeled separately. After the dimensionless products of each PD indicator quantity was calculated, the corresponding model was formulated.

\subsection{Derivation of the PD current model}

First, we explored the dependence of the PD current on the six independent variables. Using (6), we obtained the dimen- 
Table 1. Dimensions of GIS quantities to be inter-related by DA

\begin{tabular}{llll}
\hline Quantity & Symbol & Unit & Dimension \\
\hline PD current & $I_{P D}$ & $\mathrm{~A}$ & $M^{0} L^{0} T^{0} I^{1}$ \\
PD charge & $Q_{P D}$ & $\mathrm{C}$ & $M^{0} L^{0} T^{1} I^{1}$ \\
PD energy & $W_{P D}$ & $\mathrm{~J}$ & $M^{1} L^{2} T^{-2} I^{0}$ \\
Applied voltage & $V$ & $\mathrm{~V}$ & $M^{1} L^{2} T^{-3} I^{-1}$ \\
SF 6 pressure & $P$ & $\mathrm{~Pa}$ & $M^{1} L^{-1} T^{-2} I^{0}$ \\
Particle length & $L$ & $\mathrm{~m}$ & $M^{0} L^{1} T^{0} I^{0}$ \\
Particle position relative to grounded & $H_{p}$ & $\mathrm{~m}$ & $M^{0} L^{1} T^{0} I^{0}$ \\
$\quad$ electrode & & & \\
Gap between electrodes & $H_{S}$ & $\mathrm{~m}$ & $M^{0} L^{1} T^{0} I^{0}$ \\
Duration of voltage application & $t$ & $\mathrm{~s}$ & $M^{0} L^{0} T^{1} I^{0}$ \\
\hline
\end{tabular}

sionless product

$$
\pi_{I}=k I_{P D}^{a_{1}} V^{b_{1}} P^{c_{1}} L^{d_{1}} H_{p}^{e_{1}} H_{s}^{f_{1}} t^{g_{1}}
$$

or

$$
\left[\pi_{I}\right]=\left[I_{P D}\right]^{a_{1}}[V]^{b_{1}}[P]^{c_{1}}[L]^{d_{1}}\left[H_{p}\right]^{e_{1}}\left[H_{s}\right]^{f_{1}}[t]^{g_{1}}
$$

where $\left[\pi_{I}\right]=1$. Substituting the dimension of each quantity into (18), we obtained

$$
\begin{aligned}
M^{0} L^{0} T^{0} I^{0}= & \left(M^{0} L^{0} T^{0} I^{1}\right)^{a_{1}}\left(M^{1} L^{2} T^{-3} I^{-1}\right)^{b_{1}}\left(M^{1} L^{-1} T^{-2} I^{0}\right)^{c_{1}} \\
& \left(M^{0} L^{1} T^{0} I^{0}\right)^{d_{1}}\left(M^{0} L^{1} T^{0} I^{0}\right)^{e_{1}}\left(M^{0} L^{1} T^{0} I^{0}\right)^{f_{1}} \\
& \left(M^{0} L^{0} T^{1} I^{0}\right)^{g_{1}}
\end{aligned}
$$

or, equivalently

$$
M^{0} L^{0} T^{0} I^{0}=M^{b_{1}+c_{1}} L^{2 b_{1}-c_{1}+d_{1}+e_{1}+f_{1}} T^{-3 b_{1}-2 c_{1}+g_{1}} I^{a_{1}-b_{1}}
$$

The product $\pi_{I}$ was dimensionless if the following scalar equations were satisfied simultaneously

$$
\begin{aligned}
& b_{1}+c_{1}=0 \\
& 2 b_{1}-c_{1}+d_{1}+e_{1}+f_{1}=0 \\
& -3 b_{1}-2 c_{1}+g_{1}=0 \\
& a_{1}-b_{1}=0
\end{aligned}
$$

Equations (21) can be converted into the following matrix form,

$$
\left[\begin{array}{ccccccc}
0 & 1 & 1 & 0 & 0 & 0 & 0 \\
0 & 2 & -1 & 1 & 1 & 1 & 0 \\
0 & -3 & -2 & 0 & 0 & 0 & 1 \\
1 & -1 & 0 & 0 & 0 & 0 & 0
\end{array}\right]\left[\begin{array}{l}
a_{1} \\
b_{1} \\
c_{1} \\
d_{1} \\
e_{1} \\
f_{1} \\
g_{1}
\end{array}\right]=\left[\begin{array}{l}
0 \\
0 \\
0 \\
0
\end{array}\right]
$$

The matrix equation (22) can be written immediately from Table 1. Each matrix vector is a listing of the dimensional exponents for the quantity it designates. At this initial stage, each matrix entry is a small integer. Equation (22) can be further re-configured into the following form,

$$
\underbrace{\left[\begin{array}{ccccccc}
1 & 0 & 0 & 0 & 0 & 0 & 1 \\
-1 & 1 & 0 & 0 & 1 & 1 & 2 \\
-2 & 0 & 1 & 0 & 0 & 0 & -3 \\
0 & 0 & 0 & 1 & 0 & 0 & -1
\end{array}\right]}_{\text {dimensional matrix }}\left[\begin{array}{l}
c_{1} \\
d_{1} \\
g_{1} \\
a_{1} \\
e_{1} \\
f_{1} \\
b_{1}
\end{array}\right]=\left[\begin{array}{l}
0 \\
0 \\
0 \\
0
\end{array}\right]
$$

The required dimensional matrix has been constructed in (23), in which column interchanging was used so that the 4-by-4 matrix formed by the first four columns resembled an identity matrix as much as possible. We did not insist on selecting $a_{1}$ as a regime index from the outset, though ultimately we wanted it to be so. From (23), it is known according to (9) that

$$
\mathbf{A}_{1}=\left[\begin{array}{cccc}
1 & 0 & 0 & 0 \\
-1 & 1 & 0 & 0 \\
-2 & 0 & 1 & 0 \\
0 & 0 & 0 & 1
\end{array}\right] ; \quad \mathbf{B}_{1}=\left[\begin{array}{ccc}
0 & 0 & 1 \\
1 & 1 & 2 \\
0 & 0 & -3 \\
0 & 0 & -1
\end{array}\right]
$$

Subsequent operations based on each of the three methods described in the previous section are presented as follows.

\subsection{1. $\pi_{i}$ computation with method 1}

To perform the required transformation shown in Figure 2, we applied Gauss-Jordan elimination (using row interchange if needed to avoid pivoting on a zero value). The detailed elementary row operations for this elimination are explicitly shown in the left margin of Table 2. To avoid the trouble caused by the conventional layout in (9a), this table does not write the vector of indices as a column vector to the right of the dimensional matrix, but writes it as a row vector on top of it, a trick that considerably facilitates the visual reading of the scalar equivalent of a matrix equation [34]. Table 2 also omits the equality sign in (5) and adds the zero vector in the R.H.S. of (5) as an extra vector for the dimensional matrix resulting in what is called an augmented dimensional matrix [30]. The layout in Table 2 effectively resembles the way a matrix equation is stored in a computing machine.

Table 2. Application of Gauss-Jordan elimination in the DA for the PD current

\begin{tabular}{r|ccccccc|c|}
\multicolumn{1}{c}{} & $c_{1}$ & $d_{1}$ & $g_{1}$ & $a_{1}$ & $e_{1}$ & $f_{1}$ & $b_{1}$ & \\
\hline$(1)$ & $\mathbf{1}$ & 0 & 0 & 0 & 0 & 0 & 1 & 0 \\
$(2)$ & -1 & $\mathbf{1}$ & 0 & 0 & 1 & 1 & 2 & 0 \\
$(3)$ & -2 & 0 & $\mathbf{1}$ & 0 & 0 & 0 & -3 & 0 \\
$(4)$ & 0 & 0 & 0 & $\mathbf{1}$ & 0 & 0 & -1 & 0 \\
\hline$(1)$ & $\mathbf{1}$ & 0 & 0 & 0 & 0 & 0 & 1 & 0 \\
$(2)+(1)$ & 0 & $\mathbf{1}$ & 0 & 0 & 1 & 1 & 3 & 0 \\
$(3)+2(1)$ & 0 & 0 & $\mathbf{1}$ & 0 & 0 & 0 & -1 & 0 \\
$(4)$ & 0 & 0 & 0 & $\mathbf{1}$ & 0 & 0 & -1 & 0 \\
\hline
\end{tabular}

From Table 2, it is observed that no all-0 row is generated. This observation revealed that the rank of the dimensional matrix in (23) was indeed 4. Therefore, $r=p=4$. Applying (10), we obtained

$$
\begin{aligned}
& c_{1}=b_{1} \\
& d_{1}=-e_{1}-f_{1}-3 b_{1} \\
& g_{1}=b_{1} \\
& a_{1}=b_{1}
\end{aligned}
$$

Substituting back the values of $c_{1}, d_{1}, g_{1}, a_{1}$ into (17) yields

$$
\pi_{I}=k I_{P D}^{b_{1}} V^{b_{1}} P^{-b_{1}} L^{-e_{1}-f_{1}-3 b_{1}} H_{p}^{e_{1}} H_{s}^{f_{1}} t^{b_{1}}
$$

or

$$
\pi_{I}=k\left(\frac{H_{p}}{L}\right)^{e_{1}}\left(\frac{H_{s}}{L}\right)^{f_{1}}\left(\frac{I_{P D} V t}{P L^{3}}\right)^{b_{1}}
$$


Thus

$$
\pi_{I 1}=\frac{H_{p}}{L}, \quad \pi_{I 2}=\frac{H_{s}}{L}, \quad \pi_{I 3}=\frac{I_{P D} V t}{P L^{3}}
$$

We were fortunate that the output variable $I_{P D}$ appeared in a single dimensionless product at no extra manipulation cost. Otherwise, we would replace the three independent dimensional products in (28) by three others, which were still independent, but with the appearance of $I_{P D}$ confined to one of them.

\subsection{2. $\pi_{i}$ computation with method 2}

Our particular arrangement for the columns of $\mathbf{A}_{1}$ paid off herein elegantly. Since $\mathbf{A}_{1}$ was a lower triangular matrix of unit diagonal entries, its inverse $\mathbf{A}_{1}^{-1}$ was also lower triangular of unit diagonal entries, and it inherited all zero entries from $\mathbf{A}_{1}$. Only two elements in $\mathbf{A}_{1}^{-1}$ remained to be determined trivially by inspection, so that $\mathbf{A}_{1}^{-1}$ and the resulting $\mathbf{D}_{1}$ were given finally as

$$
\mathbf{A}_{1}=\left[\begin{array}{llll}
1 & 0 & 0 & 0 \\
1 & 1 & 0 & 0 \\
2 & 0 & 1 & 0 \\
0 & 0 & 0 & 1
\end{array}\right], \quad \mathbf{D}_{1}=\mathbf{A}_{1}^{-1} \mathbf{B}_{1}=\left[\begin{array}{ccc}
0 & 0 & 1 \\
1 & 1 & 3 \\
0 & 0 & -1 \\
0 & 0 & -1
\end{array}\right]
$$

As shown in Figure 3, matrix $\mathbf{C}_{1}$ was computed as follows.

$$
\mathbf{C}_{1}=\left(-\mathbf{I}_{r \times r} \mathbf{D}_{1}\right)^{T}=\left[\begin{array}{cccc}
0 & -1 & 0 & 0 \\
0 & -1 & 0 & 0 \\
-1 & -3 & 1 & 1
\end{array}\right]
$$

Using (15), we calculated the dimensionless products from (30) and recorded the resulting expressions as

$$
\pi_{I 1}=\frac{H_{p}}{L}, \quad \pi_{I 2}=\frac{H_{s}}{L}, \quad \pi_{I 3}=\frac{I_{P D} V t}{P L^{3}}
$$

which were exactly the same as those in (28).

\subsection{3. $\pi_{i}$ computation with method 3}

In method 3 , matrix $\mathbf{C}_{1}$ was computed directly using (16). For $r=p$,

$$
\mathbf{C}_{1}=\left(\mathbf{A}_{1}^{-1}\left(-\mathbf{B}_{1}\right)\right)^{T}=\left[\begin{array}{cccc}
0 & -1 & 0 & 0 \\
0 & -1 & 0 & 0 \\
-1 & -3 & 1 & 1
\end{array}\right]
$$

Since the resulting matrix was exactly the same as that in (30), the dimensionless products $\pi_{I 1}, \pi_{I 2}$, and $\pi_{I 3}$ were also exactly the same as those in (28).

After all dimensionless products were calculated, the task left was to formulate a model of the PD current. The PD current $I_{P D}$ was embedded in $\pi_{I 3}$ only. Therefore, we wrote

$$
\pi_{I 3}=k_{1} \Phi\left(\pi_{I 2}, \pi_{I 3}\right)
$$

By substitution of (28) into (32), we obtained the model of $I_{P D}$ as follows,

$$
I_{P D}=\frac{k_{1} P L^{3}}{V t} \Phi\left(\frac{H_{p}}{L}, \frac{H_{s}}{L}\right)
$$

where $k_{1}$ is an unknown dimensionless constant, and $\Phi$ is an unknown function. Further experiments might aid in determining these two unknowns.

\subsection{Derivation of the PD charge model}

In a way similar to that of the derivation of the PD current model, the PD charge derivation was started by formulating a $\pi$ equation dictated by (6) as follows,

$$
\pi_{Q}=k Q_{P D}^{a_{2}} V^{b_{2}} P^{c_{2}} L^{d_{2}} H_{p}^{e_{2}} H_{s}^{f_{2}} t^{g_{2}}
$$

or

$$
1=\left[\pi_{Q}\right]=\left[Q_{P D}\right]^{a_{2}}[V]^{b_{2}}[P]^{c_{2}}[L]^{d_{2}}\left[H_{p}\right]^{e_{2}}\left[H_{S}\right]^{f_{2}}[t]^{g_{2}}
$$

Substituting the dimensions of each quantity into (35) results in

$$
\begin{aligned}
M^{0} L^{0} T^{0} I^{0}= & \left(M^{0} L^{0} T^{1} I^{1}\right)^{a_{2}}\left(M^{1} L^{2} T^{-3} I^{-1}\right)^{b_{2}}\left(M^{1} L^{-1} T^{-2} I^{0}\right)^{c_{2}} \\
& \left(M^{0} L^{1} T^{0} I^{0}\right)^{d_{2}}\left(M^{0} L^{1} T^{0} I^{0}\right)^{e_{2}}\left(M^{0} L^{1} T^{0} I^{0}\right)^{f_{2}} \\
& \left(M^{0} L^{0} T^{1} I^{0}\right)^{g_{2}}
\end{aligned}
$$

or, equivalently

$$
\begin{aligned}
M^{0} L^{0} T^{0} I^{0}= & M^{b_{2}+c_{2}} L^{2 b_{2}-c_{2}+d_{2}+e_{2}+f_{2}} T^{a_{2}-3 b_{2}-2 c_{2}+g_{2}} \\
& I^{a_{2}-b_{2}}
\end{aligned}
$$

The product $\pi_{Q}$ is dimensionless if the following four equations are satisfied.

$$
\begin{aligned}
& b_{2}+c_{2}=0 \\
& 2 b_{2}-c_{2}+d_{2}+e_{2}+f_{2}=0 \\
& a_{2}-3 b_{2}-2 c_{2}+g_{2}=0 \\
& a_{2}-b_{2}=0
\end{aligned}
$$

Equations (38a) and (38d) are similar to (21a) and (21b), respectively.

The dimensional matrix for the current case is shown in the following matrix equation,

$$
\underbrace{\left[\begin{array}{ccccccc}
1 & 0 & 0 & 1 & 0 & 0 & 0 \\
-1 & 1 & 0 & 2 & 0 & 1 & 1 \\
-2 & 0 & 1 & -3 & 1 & 0 & 0 \\
0 & 0 & 0 & -1 & 1 & 0 & 0
\end{array}\right]}_{\text {dimensional matrix }}\left[\begin{array}{l}
c_{2} \\
d_{2} \\
g_{2} \\
b_{2} \\
a_{2} \\
e_{2} \\
f_{2}
\end{array}\right]=\left[\begin{array}{l}
0 \\
0 \\
0 \\
0
\end{array}\right]
$$

The submatrices $\mathbf{A}_{2}$ and $\mathbf{B}_{2}$ extracted from the dimensional matrix in (39) are

$$
\mathbf{A}_{2}=\left[\begin{array}{cccc}
1 & 0 & 0 & 1 \\
-1 & 1 & 0 & 2 \\
-2 & 0 & 1 & -3 \\
0 & 0 & 0 & -1
\end{array}\right] ; \quad \mathbf{B}_{2}=\left[\begin{array}{ccc}
0 & 0 & 0 \\
0 & 1 & 1 \\
1 & 0 & 0 \\
1 & 0 & 0
\end{array}\right]
$$

As in the derivation of the PD current model, we presented here three methods for computing the dimensional products $\pi_{i}$.

\subsection{1. $\pi_{i}$ computation with method 1}

The Gauss-Jordan elimination procedure for the dimensional matrix in (39) is shown in Table 3. 
Table 3. Application of Gauss-Jordan elimination in the DA for the PD charge

\begin{tabular}{r|ccccccc|c}
\multicolumn{1}{c}{} & $c_{2}$ & $d_{2}$ & $g_{2}$ & $b_{2}$ & $a_{2}$ & $e_{2}$ & $f_{2}$ & \\
\hline$(1)$ & $\mathbf{1}$ & 0 & 0 & 1 & 0 & 0 & 0 & 0 \\
$(2)$ & -1 & $\mathbf{1}$ & 0 & 2 & 0 & 1 & 1 & 0 \\
$(3)$ & -2 & 0 & $\mathbf{1}$ & -3 & 1 & 0 & 0 & 0 \\
$(4)$ & 0 & 0 & 0 & -1 & 1 & 0 & 0 & 0 \\
\hline$(1)$ & $\mathbf{1}$ & 0 & 0 & 1 & 0 & 0 & 0 & 0 \\
$(2)+(1)$ & 0 & $\mathbf{1}$ & 0 & 3 & 0 & 1 & 1 & 0 \\
$(3)+2(1)$ & 0 & 0 & $\mathbf{1}$ & -1 & 1 & 0 & 0 & 0 \\
$(4)$ & 0 & 0 & 0 & -1 & 1 & 0 & 0 & 0 \\
\hline$(1)+(4)$ & $\mathbf{1}$ & 0 & 0 & 0 & 1 & 0 & 0 & 0 \\
$(2)+3(4)$ & 0 & $\mathbf{1}$ & 0 & 0 & 3 & 1 & 1 & 0 \\
$(3)-(4)$ & 0 & 0 & $\mathbf{1}$ & 0 & 0 & 0 & 0 & 0 \\
$-(4)$ & 0 & 0 & 0 & $\mathbf{1}$ & -1 & 0 & 0 & 0 \\
\hline
\end{tabular}

From Table 3, it is known that the rank of the dimensional matrix in (39) is 4 . Therefore, $r=p=4$. The application of (10) resulted in

$$
\begin{aligned}
& c_{2}=-a_{2} \\
& d_{2}=-3 a_{2}-e_{2}-f_{2} \\
& g_{2}=0 \\
& b_{2}=a_{2}
\end{aligned}
$$

Substituting back the values of $c_{2}, d_{2}, g_{2}, b_{2}$ into (34) yields

$$
\pi_{Q}=k Q_{P D}^{a_{2}} V^{a_{2}} P^{-a_{2}} L^{-3 a_{2}-e_{2}-f_{2}} H_{p}^{e_{2}} H_{s}^{f_{2}}
$$

or

$$
\pi_{Q}=k\left(\frac{Q_{P D} V}{P L^{3}}\right)^{a_{2}}\left(\frac{H_{p}}{L}\right)^{e_{2}}\left(\frac{H_{s}}{L}\right)^{f_{2}}
$$

Thus

$$
\pi_{Q 1}=\frac{Q_{P D} V}{P L^{3}}, \quad \pi_{Q 2}=\frac{H_{p}}{L}, \quad \pi_{Q 3}=\frac{H_{s}}{L}
$$

\subsection{2. $\pi_{i}$ computation with method 2}

The inverse of the matrix $\mathbf{A}_{2}$ in (40) is also easy to obtain, most conveniently by applying the same elementary row operations in Table 3 to $\mathbf{I}_{4 \times 4}$. Along with $\mathbf{D}_{2}$, it is

$$
\mathbf{A}_{2}=\left[\begin{array}{cccc}
1 & 0 & 0 & 1 \\
1 & 1 & 0 & 3 \\
2 & 0 & 1 & -1 \\
0 & 0 & 0 & -1
\end{array}\right], \quad \mathbf{D}_{2}=\mathbf{A}_{2}^{-1} \mathbf{B}_{2}=\left[\begin{array}{ccc}
1 & 0 & 0 \\
3 & 1 & 1 \\
0 & 0 & 0 \\
-1 & 0 & 0
\end{array}\right]
$$

As shown in Figure 3, matrix $\mathbf{C}_{2}$ was computed as follows.

$$
\mathbf{C}_{2}=\left(-\mathbf{I}_{r \times r} \mathbf{D}_{2}\right)^{T}=\left[\begin{array}{cccc}
-1 & -3 & 0 & 1 \\
0 & -1 & 0 & 0 \\
0 & -1 & 0 & 0
\end{array}\right]
$$

Using (15), the dimensionless products were calculated from (46) with the resulting expressions as follows.

$$
\pi_{Q 1}=\frac{Q_{P D} V}{P L^{3}}, \quad \pi_{Q 2}=\frac{H_{p}}{L}, \quad \pi_{Q 3}=\frac{H_{s}}{L}
$$

which were exactly the same as those in (44).

\subsection{3. $\pi_{i}$ computation with method 3}

In method 3, matrix $\mathbf{C}_{2}$ was computed directly using (16). For $r=p$,

$$
\mathbf{C}_{2}=\left(\mathbf{A}_{2}^{-1}\left(-\mathbf{B}_{2}\right)\right)^{T}=\left[\begin{array}{cccc}
-1 & -3 & 0 & 0 \\
0 & -1 & 0 & 0 \\
0 & -1 & 0 & 0
\end{array}\right]
$$

Since the resulting matrix was exactly the same as that in (46), the dimensionless products $\pi_{Q 1}, \pi_{Q 2}$, and $\pi_{Q 3}$ were also exactly the same as those in (44).

Because the PD charge $Q_{P D}$ was embedded solely in $\pi_{Q 1}$, we could conveniently write

$$
\pi_{Q 1}=k_{2} \varphi\left(\pi_{Q 2}, \pi_{Q 3}\right)
$$

By substitution of (44) into (48), the model of the PD charge results as

$$
Q_{P D}=\frac{k_{2} P L^{3}}{V} \varphi\left(\frac{H_{p}}{L}, \frac{H_{s}}{L}\right)
$$

where $k_{2}$ is an unknown dimensionless constant, and $\varphi$ is an unknown function. Again, further experiments might aid in determining these two unknowns.

\subsection{Derivation of the PD energy model}

The third PD-related quantity to be modeled herein is the energy released during PD occurrence. The $\pi$ expression for the PD energy, as dictated by (6) and with the utilization of data in Table 1, is given as follows.

$$
\pi_{W}=k W_{P D}^{a_{3}} V^{b_{3}} P^{c_{3}} L^{d_{3}} H_{p}^{e_{3}} H_{s}^{f_{3}} t^{g_{3}}
$$

or

$$
\left[\pi_{W}\right]=\left[W_{P D}\right]^{a_{3}}[V]^{b_{3}}[P]^{c_{3}}[L]^{d_{3}}\left[H_{p}\right]^{e_{3}}\left[H_{S}\right]^{f_{3}}[t]^{g_{3}}
$$

With $\left[\pi_{W}\right]=1$ and the dimensions of each quantity in the righthand side of (51) being substituted for by the values in Table 1, the following expression is obtained,

$$
\begin{aligned}
M^{0} L^{0} T^{0} I^{0}= & M^{a_{3}+b_{3}+c_{3}} L^{2 a_{3}+2 b_{3}-c_{3}+d_{3}+e_{3}+f_{3}} \\
& T^{-2 a_{3}-3 b_{3}-2 c_{3}+g_{3}} I^{-b_{3}}
\end{aligned}
$$

Based on (52), the product $\pi_{W}$ could be rendered dimensionless if the following set of equations was simultaneously met,

$$
\begin{aligned}
& a_{3}+b_{3}+c_{3}=0 \\
& 2 a_{3}+2 b_{3}-c_{3}+d_{3}+e_{3}+f_{3}=0 \\
& -2 a_{3}-3 b_{3}-2 c_{3}+g_{3}=0 \\
& -b_{3}=0
\end{aligned}
$$

The matrix equation for (53) is presented as follows.

$$
\underbrace{\left[\begin{array}{ccccccc}
1 & 0 & 0 & 1 & 1 & 0 & 0 \\
2 & 1 & 0 & 2 & -1 & 1 & 1 \\
-2 & 0 & 1 & -3 & -2 & 0 & 0 \\
0 & 0 & 0 & -1 & 1 & 0 & 0
\end{array}\right]}_{\text {dimensional matrix }}\left[\begin{array}{l}
a_{3} \\
d_{3} \\
g_{3} \\
b_{3} \\
c_{3} \\
e_{3} \\
f_{3}
\end{array}\right]=\left[\begin{array}{l}
0 \\
0 \\
0 \\
0
\end{array}\right]
$$


The sub-matrices $\mathbf{A}_{3}$ and $\mathbf{B}_{3}$ are

$$
\mathbf{A}_{3}=\left[\begin{array}{cccc}
1 & 0 & 0 & 1 \\
2 & 1 & 0 & 2 \\
-2 & 0 & 1 & -3 \\
0 & 0 & 0 & -1
\end{array}\right] ; \quad \mathbf{B}_{3}=\left[\begin{array}{ccc}
1 & 0 & 0 \\
-1 & 1 & 1 \\
-2 & 0 & 0 \\
0 & 0 & 0
\end{array}\right]
$$

As in the derivation of the PD current and the PD charge models, we also implemented our three methods for computing the dimensional products $\pi_{i}$.

\subsection{1. $\pi_{i}$ computation with method 1}

The Gauss-Jordan elimination procedure for the dimensional matrix in (54) is shown in Table 4.

Table 4. Application of Gauss-Jordan elimination in the DA for the PD energy

\begin{tabular}{r|ccccccc|c|}
\multicolumn{1}{c}{} & $a_{3}$ & $d_{3}$ & $g_{3}$ & $b_{3}$ & $c_{3}$ & $e_{3}$ & $f_{3}$ & \\
\hline$(1)$ & $\mathbf{1}$ & 0 & 0 & 1 & 1 & 0 & 0 & 0 \\
$(2)$ & 2 & $\mathbf{1}$ & 0 & 2 & -1 & 1 & 1 & 0 \\
$(3)$ & -2 & 0 & $\mathbf{1}$ & -3 & -2 & 0 & 0 & 0 \\
$(4)$ & 0 & 0 & 0 & -1 & 0 & 0 & 0 & 0 \\
\hline$(1)$ & $\mathbf{1}$ & 0 & 0 & 1 & 1 & 0 & 0 & 0 \\
$(2)-2(1)$ & 0 & $\mathbf{1}$ & 0 & 0 & -3 & 1 & 1 & 0 \\
$(3)+2(1)$ & 0 & 0 & $\mathbf{1}$ & -1 & 0 & 0 & 0 & 0 \\
$-(4)$ & 0 & 0 & 0 & $\mathbf{1}$ & 0 & 0 & 0 & 0 \\
\hline$(1)-(4)$ & $\mathbf{1}$ & 0 & 0 & 0 & 1 & 0 & 0 & 0 \\
$(2)$ & 0 & $\mathbf{1}$ & 0 & 0 & 3 & 1 & 1 & 0 \\
$(3)+(4)$ & 0 & 0 & $\mathbf{1}$ & 0 & 0 & 0 & 0 & 0 \\
$(4)$ & 0 & 0 & 0 & $\mathbf{1}$ & 0 & 0 & 0 & 0
\end{tabular}

From Table 4, the rank of the dimensional matrix in (54) is again 4 . Therefore, $r=p=4$. The application of (10) yielded

$$
\begin{aligned}
& a_{3}=-c_{3} \\
& d_{3}=3 c_{3}-e_{3}-f_{3} \\
& g_{3}=0 \\
& b_{3}=0
\end{aligned}
$$

Substituting back the values of $a_{3}, d_{3}, g_{3}, b_{3}$ into (50) yielded

$$
\pi_{W}=k W_{P D}^{-c_{3}} P^{c_{3}} L^{3 c_{3}-e_{3}-f_{3}} H_{p}^{e_{3}} H_{s}^{f_{3}}
$$

or

$$
\pi_{W}=k\left(\frac{P L^{3}}{W_{P D}}\right)^{c_{3}}\left(\frac{H_{p}}{L}\right)^{e_{3}}\left(\frac{H_{s}}{L}\right)^{f_{3}}
$$

Thus

$$
\pi_{W 1}=\frac{P L^{3}}{W_{P D}}, \quad \pi_{W 2}=\frac{H_{p}}{L}, \quad \pi_{W 3}=\frac{H_{s}}{L}
$$

\subsection{2. $\pi_{i}$ computation with method 2}

The inverse of the matrix $\mathbf{A}_{3}$ in (55) was again easy to obtain, most conveniently by applying the same elementary row operations in Table 4 to the unit matrix $\mathbf{I}_{4 \times 4}$. Along with $\mathbf{D}_{3}$, it is

$$
\mathbf{A}_{3}=\left[\begin{array}{cccc}
1 & 0 & 0 & 1 \\
-2 & 1 & 0 & 0 \\
2 & 0 & 1 & -1 \\
0 & 0 & 0 & -1
\end{array}\right], \quad \mathbf{D}_{3}=\mathbf{A}_{3}^{-1} \mathbf{B}_{3}=\left[\begin{array}{ccc}
1 & 0 & 0 \\
-3 & 1 & 1 \\
0 & 0 & 0 \\
0 & 0 & 0
\end{array}\right]
$$

As shown in Figure 3, matrix $\mathbf{C}_{3}$ was computed as follows.

$$
\mathbf{C}_{3}=\left(-\mathbf{I}_{r \times r} \mathbf{D}_{3}\right)^{T}=\left[\begin{array}{cccc}
-1 & -3 & 0 & 0 \\
0 & -1 & 0 & 0 \\
0 & -1 & 0 & 0
\end{array}\right]
$$

Based on (15), the dimensionless products were calculated from (61) with the following resulting expressions.

$$
\pi_{W 1}=\frac{P L^{3}}{W_{P D}}, \quad \pi_{W 2}=\frac{H_{p}}{L}, \quad \pi_{W 3}=\frac{H_{s}}{L}
$$

which were exactly the same as those in (59).

\subsection{3. $\pi_{i}$ computation with method 3}

For $r=p$, matrix $\mathbf{C}_{3}$ is computed as follows.

$$
\mathbf{C}_{3}=\left(\mathbf{A}_{3}^{-1}\left(-\mathbf{B}_{3}\right)\right)^{T}=\left[\begin{array}{cccc}
-1 & -3 & 0 & 0 \\
0 & -1 & 0 & 0 \\
0 & -1 & 0 & 0
\end{array}\right]
$$

Since the resulting matrix was exactly the same as that in (61), the dimensionless products $\pi_{W 1}, \pi_{W 2}$, and $\pi_{W 3}$ were also exactly the same as those in (59).

The relationship among $\pi_{W 1}, \pi_{W 2}$, and $\pi_{W 3}$ is expressed below,

$$
\pi_{W 1}=k_{3} \chi\left(\pi_{W 2}, \pi_{W 3}\right)
$$

Substituting (59) into (63) resulted in

$$
W_{P D}=\frac{P L^{3}}{k_{3} \chi\left(\frac{H_{p}}{L}, \frac{H_{s}}{L}\right)}
$$

where $k_{3}$ is an unknown dimensionless constant, and $\chi$ is an unknown function. Again, further experiments might aid in determining these two unknowns.

\subsection{Discussion on the derived models}

Although DA per se does not produce complete models of the PD quantities, as further experiments are required, we can still deduce a wealth of qualitative information from (33), (49), and (64) [30, 31]. Equations (33), (49), and (64) assert the adverse effect of particle length $L$ on the GIS. This length is not only directly proportional to each of the three PD quantities studied in this paper, namely $I_{P D}, Q_{P D}$, and $W_{P D}$, but each of these PD quantities is even directly proportional to $L^{3}$, indicating any increase in $L$ corresponds to a higher increase in each of them. For example, if $L$ increases by a factor 2 , then each of the PD quantities increases by a factor of 8 , of course provided other variables are kept constant.

A careful observation should be made on $P$ and $V$ in (33) and (49), and on $P$ in (64). In (33) and (49), it seems illogical that $P$ is directly proportional and $V$ is inversely proportional to the respective $\mathrm{PD}$ quantities. In fact, when the $\mathrm{SF}_{6}$ pressure $P$ is increased (until a certain limit), its dielectric strength gets higher and, consequently, the PD development can be suppressed. Conversely, higher applied voltage $V$ results in stronger PD development. To compromise with this, the term $P / V$ in (33) and (49) and the term $V$ in (64) should be deemed as constants, rather than as determining variables.

Further observation is also carried out in (49). In retrospect, we might have avoided the detailed derivations in Section 3.2 if we are in a position to conjecture that the PD current is simply the PD charge divided by the duration of voltage application. Based on this conjecture, equation (33) can effectively lead to equation (49). The DA role stops here, and we face two possible courses of action, namely (a) the DA conjecture is a proven fact, 
so that DA just confirms what is already known, and (b) the DA conjecture is just a dimensionally correct proposition that is still in need of an analytical proof. To the best of our knowledge, this issue is still an open question that warrants further investigation.

In passing, we stress that we deliberately employed distinct symbols $\Phi, \varphi$ and $\chi$ for the functions in expressions (33), (49), and (64). Our purpose is to abide by DA considerations and allow the designated functions to be different, albeit we do not rule out the possibility that they might be the same. In fact, past experience indicated that these three symbols were more likely to express the same function rather than to designate different functions. We based the aforementioned DA conjecture on the assumption that the two functions $\Phi$ and $\varphi$ are the same.

\section{Conclusion}

In this paper, dimensional analysis has been carried out to develop the models of PDs initiated by a metallic particle in a GIS. The quantities modeled included the PD current, the PD charge, and the PD energy. From past experiments, it is suggested that each of these quantities is dependent on several other independent quantities including the $\mathrm{SF}_{6}$ pressure, the applied voltage level, the particle length, the particle position, the voltage application duration, and the spacer height. Thus, the PD models were built in terms of these independent quantities. The role of dimensional analysis for the modeling task is to compute a new set of fewer parameters, called as the dimensionless products, which replace the original variables. Three methods based on matrix operations have been used for this purpose in each of the three PD quantities. The precisely same results have been obtained from these methods. The calculated dimensional products were then utilized to formulate models for the PD current, PD charge, and PD energy. These models are not only useful for engineering utility, but also for some theoretical work for their verification.

\section{References}

1. P. Bolin and H. Koch, Gas insulated substation GIS, IEEE Power Engineering Society General Meeting, Montreal, QC, Canada, 2006, pp. 1-3.

2. H. S. Jain. Gas-Insulated Sub-Station/Switchgear (GIS). in Handbook of Switchgears, S. R. K. Shukla, S. Indrapal, and S. S. G. Deshpande, Eds. New York, NY: The McGraw-Hill Companies, 2007.

3. M. A. Haseeb and M. J. Thomas, Disconnector switching induced transient voltage and radiated fields in a $1100 \mathrm{kV}$ gas insulated substation, Electr. Power Syst. Res. 161 (2018) 86-94.

4. M. M. Morcos, S. A. Ward, H. Anis, K. D. Srivastava, and S. M. Gubanski, Insulation integrity of GIS / GITL systems and management of particle contamination, IEEE Electr. Insul. Mag., 16 (2000) 25-37.

5. I. A. Metwally, Status review on partial discharge measurement techniques in gas-insulated switchgear/lines, Electr. Power Syst. Res. 69 (2004) 25-36.

6. H. Okubo, K. Nishizawa, T. Okusu, N. Hayakawa, and F. Endo, Partial discharge detection techniques under the Condition of metallic particle adhering to solid spacer in SF6, Electrical Insulation and Dielectric Phenomena, Ann. Rep. Conf., Quebec, QC, Canada, 2008, pp. 395-399.

7. F. N. Budiman, Y. Khan, A. A. Khan, A. Beroual, and N. H. Malik, Dependence of particle initiated PD characteristics on size and position of metallic particle adhering to the spacer surface in GIS, Int. J. Electr. Comput. Eng. 7 (2013) 349-353.

8. Y. Khan, F. N. Budiman, A. Beroual, N. H. Malik, and A. A. Al-Arainy, The estimation of size and position of contaminating particle adhering to the insulating spacer surface in gas-insulated systems, Eur. Phys. J. Appl. Phys. 62 (2013) 20801.

9. F. N. Budiman, Y. Khan, A. A. Al-Arainy, N. H. Malik, and A. Beroual,
Estimation of particle initiated PD inception voltage around spacer in GIS, Power Engineering, Energy and Electrical Drives, Int. Conf., Istanbul, Turkey, 2013, pp. 517-521.

10. F. Gu, Identification of Partial Discharge Defects in Gas-Insulated Switchgears by Using a Deep Learning Method, IEEE Access 8 (2020) 163894-163902.

11. Y. Wang, J. Yan, Z. Yang, T. Liu, Y. Zhao, and J. Li, Partial Discharge Pattern Recognition of Gas-Insulated Switchgear via a Light-Scale Convolutional Neural Network, Energies 12 (2019) 1-19.

12. V. Tuyet-Doan, H. Pho, B. Lee, and Y. Kim, Deep Ensemble Model for Unknown Partial Discharge Diagnosis in Gas-Insulated Switchgears Using Convolutional Neural Networks, IEEE Access 9 (2021) 80524-80534.

13. J. Tang, Q. Zhou, M. Tang, and Y. Xie, Study on Mathematical Model for VHF Partial Discharge of Typical Insulated Defects in GIS, IEEE Trans. Dielect. Elec. Insul. 14 (2007) 30-38.

14. Y. Wang, J. Yan, Q. Sun, J. Li, and Z. Yang, A MobileNets Convolutional Neural Network for GIS Partial Discharge Pattern Recognition in the Ubiquitous Power Internet of Things Context: Optimization, Comparison, and Application, IEEE Access 7 (2019) 150226-150236.

15. Z. Xin and Q. Jiangtao, De-noising of GIS UHF Partial Discharge Monitoring based on Wavelet Method, Proc. Env. Sci. 11 (2011) 1302-1307.

16. X. Li, Z. Wang, X. Wang, M. Rong, and D. Liu, Chromatic processing for feature extraction of PD-induced UHF signals in GIS, Glob. Ener. Intercon. 3 (2020) 494-503.

17. N. Gupta and T. S. Ramu, Estimation of Partial Discharge Parameters in GIS using Acoustic Emission Techniques, J. Sou. Vibr. 247 (2001) 243-260.

18. I. Wahyudi and A. Sakti, Analyzing the profit-loss sharing contracts with Markov model, Comm. Sci. Tech. 1 (2016) 78-88.

19. H. Xia, J. Zhang, G. Du, H. Pan, W. Duan, and X. She, Aeolian sand bearing capacity in the Mu Us Desert of China based on the California Bearing Ratio, J. Eng. Res. 8 (2020) 28-41.

20. M. Mohammadzaheri, A. Firoozfar, D. Mehrabi, M. Emadi, and A. Alqallaf, Temperature estimation for a point of an infrared dryer using temperature of neighbouring points: An artificial neural network approach, J. Eng. Res. 7 (2019) 138-150.

21. J. O. Oladigbolu and A. M. Rushdi, Investigation of the Corona Discharge Problem Based on Different Computational Approaches of Dimensional Analysis, J. Eng. Res. Rep. 15 (2020) 17-36.

22. M. A. Rushdi and A. M. Rushdi, Matrix Dimensional Analysis for Electromagnetic Quantities, Int. J. Math. Eng. Man. Sci. 6 (2021) 636-644.

23. S. M. Hoek, S. Coenen, M. Bornowski, and S. Tenbohlen, Fundamental differences of the PD measurement according to IEC 60270 and in UHF range, Condition Monitoring and Diagnosis, Int. Conf., Beijing, China, 2008, pp. 79-81.

24. E. Lemke et al., Guide for Electrical Partial Discharge Measurements on Compliance to IEC 60270, Electra 241 (2008) 60-68.

25. S. U. Haq, L. H. A. Teran, M. K. W. Stranges, and W. Veerkamp, What can go wrong during stator coil partial discharge measurements according to IEC 60270?, 11th PCIC Europe, Amsterdam, Netherlands, 2014, pp. 1-5.

26. S. U. Haq, M. K. W. Stranges, and B. Wood, Comparative study of IEC 60270 compliant instruments for partial discharge pattern acquisition, PCIC Tech. Conf. PCIC, Philadelphia, PA, USA, 2016, pp. 1-8.

27. T. Kita, Y. Uosaki, and T. Moriyoshi, Static relative permittivity of sulfur hexafluoride up to $30 \mathrm{MPa}$, Berichte der Bunsengesellschaft/Phys. Chem. 98 (1994) 112-118.

28. S.-Y. Woo, D.-H. Jeong, K.-B. Seo, and J.-H. Kim, A study on dielectric strength and insulation property of SF6/N2 mixtures for GIS, J. Int. Counc. Electr. Eng. 2 (2012) 104-109.

29. J. J. Sharp and E. Moore, A systematic approach to the development of echelon matrices for dimensional analysis, Int. J. Math. Educ. Sci. Technol. 19 (1988) 461-467.

30. M. A. Rushdi and A. M. Rushdi, On the fundamental masses derivable by dimensional analysis, J. KAU Eng. Sci. 27 (2016) 35-42.

31. R. Bhaskar and A. Nigam, Qualitative physics using dimensional analysis, Artif. Intell. 45 (1990) 73-111.

32. W. H. Middendorf, Use of Dimensional Analysis in Present Day Design Environment, IEEE Trans. Educ. E-29 (1986) 190-195.

33. J. J. Sharp and E. Moore, Partial analysis and matrix methods, Int. J. Math. Educ. Sci. Technol. 14 (1983) 393-402.

34. A. M. Rushdi, Development of modified nodal analysis into a pedagogical tool, IEEE Trans. Educ. E-28 (1985) 17-25. 